\title{
Early Discharge with Home Care Tube Feeding Program Benefits on Two Years Corrected Age Neurodevelopmental Outcomes, Breastfeeding and First Year Hospital Readmissions for Premature Infants
}

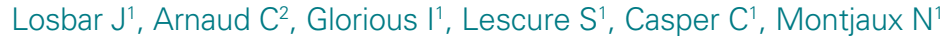 \\ ${ }^{1}$ NICU, Children's Hospital, Toulouse University Hospital, France \\ 2 UMR 1017, INSERM, Toulouse University Hospital France
}

\section{Objective}

Our objective was to determine the impact of an Early Discharge with Home Care Tube Feeding program (EDHC) - regular discharge on two-years corrected-age (CA) neurodevelopmental outcomes for preterm infants born between 25 and 35 gestational age (GA). Secondary outcome measures were length of hospitalization stay, breastfeeding and first year hospital readmissions.

\section{Methods}

This observational study compared 415 EDHC preterm infants from Toulouse University Hospital, born between 2008 and 2015, and 3186 preterm infants of the EPIPAGE 2 study cohort born in all French newborn units in 2011. Neurodevelopmental Ages and Stages Questionnaire (ASQ) was used to assess neurodevelopmental outcomes. Length of hospital stay, breastfeeding rates at discharge and six-months and hospital readmission rates during the first year were compared between the two groups. Differences in the two populations characteristics were adjusted with multivariate multilevel regression analyses.

\section{Results}

At two-years CA data on ASQ were available for $125 \mathrm{EDHC}$ and 2066 EPIPAGE 2 preterm children. EDHC preterm singletons had $61 \%$ less risk to obtain a total ASQ score below threshold of $220(\mathrm{OR}=0.39$ [0.32-0.48], $\mathrm{p}<0.001)$, less risk to have communication abilities above threshold $(\mathrm{OR}=0.42[0.34$ $0.53] \mathrm{p}<0.0001)$, fine motor skills above threshold $(\mathrm{OR}=0.63$ [0.51-0.78] $\mathrm{p}<0.0001)$, problem solving abilities above threshold $(0 \mathrm{R}=0.53[0.43-0.66] \mathrm{p}<0.0001)$, and personal-social skills above threshold $(\mathrm{OR}=0.64[0.52-0.78] \mathrm{p}<0.0001)$.

Length of hospital stay was nine days shorter for the EDHC preterm infants $(\mathrm{p}<0,0001)$.

EDHC preterm children were more likely to be breastfed at final discharge $(\mathrm{OR}=3.59$ [2.82-4.58], $\mathrm{p}<0.001$ for singletons and $\mathrm{OR}=2.25[1.62-3.14], \mathrm{p}<0.001$ for multiples), and breastfeeding was more likely to be continued over six months among those same children ( $\mathrm{OR}=1.76$ [1.34-2.32], $\mathrm{p}<0.001$ for singletons, $\mathrm{OR}=3.64$ [2.10-6.32], $\mathrm{p}<0.001$ for multiples). Finally, EDHC children singletons had less risk to be readmitted in hospital during the first year $(\mathrm{OR}=0.65$ [0.55 to 0.77$], \mathrm{p}<$ $0.001)$.

\section{Conclusion}

The Early Discharge with Home Care Program seems to improve neurodevelopmental outcome at two years of age, length of hospital stay and breastfeeding among preterm infants. It seems also to protect from hospital readmission during the first year. Home care should be promoted in newborn intensive care policies.

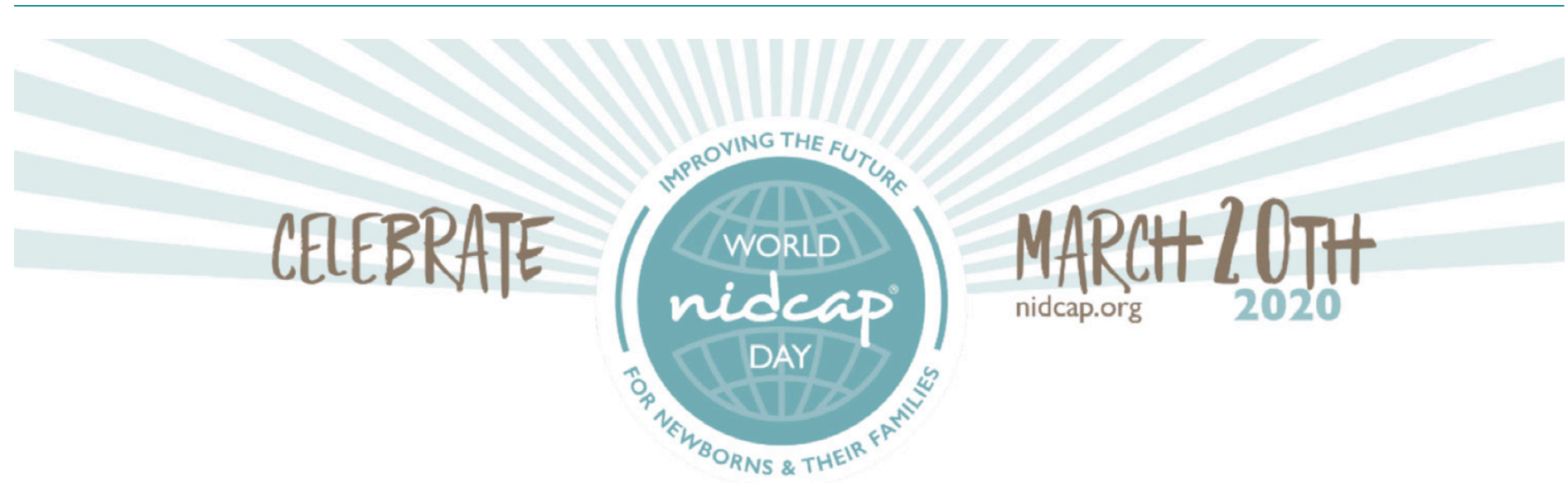

The WHO has designated 2020 the International Year of the Nurse and the Midwife. Nurses and midwives play a vital role in providing health and caring for mothers and babies. We would like to hear your stories and how NIDCAP has enhanced the role of nurses and midwives.

$8 \cdot 2020 \cdot$ Developmental Observer 